\title{
Erratum to: Abdominal spilled stones: ultrasound findings
}

F. Torello Viera, ${ }^{1}$ E. Armellini, ${ }^{1}$ L. Rosa, ${ }^{1}$ V. Ravetta, ${ }^{1}$ M. Alessiani, ${ }^{2}$ P. Dionigi, ${ }^{2}$ S. Rossi ${ }^{1}$

${ }^{1}$ Medicina VI, I.R.C.C.S. Policlinico San Matteo, Pavia, Italy

${ }^{2}$ Chirurgia Epato-pancreatica, I.R.C.C.S. Policlinico San Matteo, Pavia, Italy

Erratum to: Abdom Imaging (2006) 31:564-567 DOI 10.1007/s00261-005-0241-8

This article was inadvertently published a second time under DOI: 10.1007/s00261-006-9090-3.

Official publication is under DOI: 10.1007/s00261-005-0241-8 appearing in Abdom Imaging (2006) 31:564-567.

The online version of the original article can be found under doi:10.1007/s00261-005-0241-8.

Correspondence to: F. Torello Viera; email: f.torelloviera@smatteo. pv.it 